\title{
Le changement : I'avenir de la pharmacie
}

\author{
par Patricia Macgregor
}

E n août 2015, la Société canadienne des pharmaciens d'hôpitaux (SCPH) lancera un nouveau plan stratégique servant à guider la Société dans sa réponse aux besoins des pharmaciens d'hôpitaux et d'autres milieux de soins de santé en collaboration au cours des prochaines années. Le projet SCPH 2015 sera achevé et nous espérons que la promotion de l'excellence clinique léguée en héritage continue de croître, que l'application des connaissances et le partage des succès imprégnés dans la culture perdurent, et que l'engagement envers l'amélioration des soins et des résultats thérapeutiques des patients demeure.

La profession de pharmacien fait toujours face à d'importants défis, et ce, de l'approvisionnement en médicaments jusqu'au chevet des patients. La gestion des pénuries de médicaments a poussé l'ensemble des secteurs pharmaceutiques à coopérer plus étroitement à la prévention de futures pénuries. Le champ de pratique des pharmaciens et des techniciens en pharmacie s'est élargi à différents degrés au pays, les pharmaciens voyant leur rôle étendu à la prescription et à l'injection de médicaments, à la promotion de la santé, et à la prévention et au traitement de maladies. Des changements ont été introduits pour réglementer les pratiques en matière de préparation de produits pharmaceutiques et l'agrément des pharmacies d'hôpitaux. Les exigences relatives à la responsabilisation et aux critères de jugement se sont accrues, ce qui impose un examen minutieux de la pharmacie clinique et incite par conséquent à l'élaboration d'indicateurs de rendement validés.

De plus, on observe que les soins aux patients gagnent en complexité, une situation qui accentue la pression sur les pharmaciens, mais qui offre aussi des occasions d'apprentissage et de spécialisation dans la pratique de la pharmacie. Les modèles de pratique hospitalière, notamment, évoluent vers des modèles de soins davantage cliniques et intégrés, et l'on parle de plus en plus du besoin et du désir d'établir une certification pour la spécialisation au Canada.

Le passage au doctorat de premier cycle en pharmacie comme diplôme d'accès à la pratique pour les pharmaciens ainsi que l'augmentation des besoins en places de stage pour les étudiants qui en découlent ont une influence sur les programmes de formation en milieu hospitalier et sur les exigences envers les pharmaciens, ce qui pousse à remettre en question l'infrastructure et les approches actuelles. De nouveaux modèles de formation expérientielle dans les hôpitaux et les soins primaires sont nécessaires si l'on souhaite répondre à ces besoins croissants.

De fait, le facteur récurrent dans tout ceci est le changement : dans l'enseignement et la formation, dans la pratique clinique, dans les méthodes de travail, dans l'expertise et le champ d'exercice professionnel, et dans les normes réglementaires. Pour bien faire face au changement, il faut d'abord l'accepter. Ceci dit, l'infrastructure et les ressources représentent des catalyseurs nécessaires à la mise en place du changement, et la mesure et le compte rendu du rendement constituent des outils précieux pour communiquer l'incidence du changement.

Le projet SCPH 2015 a offert un cadre pour l'excellence en pratique et la mesure du progrès. Le Canadian National Clinical Pharmacy Key Performance Indicators Collaborative, en partenariat avec la SCPH, offrira bientôt une boîte à outils qui servira à appliquer les connaissances pour la mesure et le compte rendu des résultats. L'Association des facultés de pharmacie du Canada, appuyée en partie par le Plan directeur pour la pharmacie, met au point un programme de perfectionnement des précepteurs ainsi que des modèles de formation expérientielle qui permettront de répondre à la demande croissante de stages étudiants.

Ainsi, au cours de cette période pleine de changements et d'occasions à saisir, la SCPH continuera de soutenir ses membres en cherchant des solutions aux défis qui se profilent. À quoi ressemblera la profession de pharmacien dans l'avenir? Quels seront les besoins des pharmaciens travaillant dans les hôpitaux et les autres milieux de soins de santé en collaboration en ce qui a trait à l'éducation, aux lignes directrices, à la spécialisation et à la reconnaissance professionnelle? Quelle structure et quelle réglementation encadreront l'exercice de la pharmacie? Ces questions, ainsi que d'autres, guideront le développement du nouveau plan stratégique de la SCPH et seront au cœur des activités pour les années à venir.

Patricia Macgregor, B. Sc., R. Ph., M. R. Pharm. S., M. H. Sc., C.H.E., est présidente sortante et agente de liaison pour la vision de la Société canadienne des pharmaciens d'hôpitaux. 